\title{
Transferable single-crystal GaN thin films grown on chemical vapor-deposited hexagonal BN sheets
}

\author{
Kunook Chung ${ }^{1}$, Hongseok $\mathrm{Oh}^{1}$, Janghyun $\mathrm{Jo}^{2}$, Keundong Lee ${ }^{1}$, Miyoung Kim² and Gyu-Chul $\mathrm{Yi}^{1}$ \\ Single-crystal gallium nitride (GaN) layers were directly grown on centimeter-scale hexagonal boron nitride (h-BN). Using \\ chemical vapor deposition (CVD), centimeter-scale h-BN films were synthesized on a single-crystal Ni(111) and readily \\ transferred onto amorphous fused silica supporting substrates that had no epitaxial relationship with GaN. For growing fully \\ coalescent GaN layers on h-BN, the achievement of high-density crystal growths was a critical growth step because the \\ $s p^{2}$-bonded $\mathrm{h}$-BN layers are known to be free of dangling bonds. Unlike GaN layers grown on a typical heterogeneous sapphire \\ substrate, the morphological and microstructural results strongly suggest a high-density growth feature that is driven by the \\ atomic cliffs inherent in the CVD-grown h-BN layers. More importantly, the GaN layers grown on CVD-grown h-BN exhibited a flat \\ and continuous surface morphology with well-aligned crystal orientations both along the $c$-axis and in-plane, indicating the \\ characteristics of GaN heteroepitaxy on h-BN.
}

NPG Asia Materials (2017) 9, e410; doi:10.1038/am.2017.118; published online 28 July 2017

\section{INTRODUCTION}

There has been significant demand for the fabrication of conventional semiconductor devices in transferable and flexible forms to provide a route for the development of next-generation optoelectronics and electronics. ${ }^{1-3}$ Among the various efforts to meet this demand, a new material system has been developed that involves the growth of semiconductor materials directly on twodimensional (2D) layered graphene. Because semiconductors that are grown on graphene layers can be easily transferred to other foreign substrates, including plastic and metal, all cost restrictions arising from substrates can be avoided; ${ }^{4-7}$ furthermore, the semimetallic graphene substrates can simultaneously act as current injection and/or spreading layers. Based on these advantages, various group II-VI and III-V semiconductor nanostructures and thin films have been grown on graphene for a range of applications, including light-emitting diodes, solar cells, power generators and sensors. ${ }^{8-10}$ However, semiconductor heterostructures grown on 2D films still exhibit limited manufacturing scalability because of the difficulty of preparing large-size singlecrystalline $2 \mathrm{D}$ substrates. Although single-crystal 2D substrates can ensure that highly crystalline semiconductors can be grown for excellent, uniform device performance, small flakes of mechanically delaminated 2D layered materials are not desirable for practical device applications. In addition, the typical large-scale 2D films synthesized on metal foils by chemical vapor deposition (CVD) are polycrystalline that degrades the crystallinity of the semiconductors grown on CVD-grown 2D films. In this study, we report a method for preparing single-crystal gallium nitride (GaN) films on 2D substrates by using centimeter-scale single-crystalline CVD-grown 2D films as a growth substrate. The structural characteristics of the GaN films were investigated using X-ray diffraction (XRD) and transmission electron microscopy (TEM).

Here, we employed transferable single-crystal chemical vapordeposited hexagonal boron nitride (h-BN) to grow GaN films. Another important feature of the $2 \mathrm{D}$ substrate that has received only limited attention is its ability to be substituted between graphene and $\mathrm{h}-\mathrm{BN}$, where h-BN is a dielectric insulator, in contrast to the semimetallic graphene. The h-BN also has excellent optical transparency, mechanical strength and flexibility. ${ }^{11,12}$ Graphene and h-BN have similar structural compatibility for growing semiconductors because of their identical hexagonal crystal structures with a small lattice mismatch of $1.3 \%$. In addition, the low dielectric $2 \mathrm{D}$ h-BN material can prevent leakage current paths from a source terminal downward to substrates ${ }^{13,14}$ and enables the fabrication of electrically isolated semiconductor devices with high breakdown voltages. Accordingly, h-BN substrates can complement their graphene counterparts, thereby increasing the range of semiconductor-2D heterostructure device applications from optoelectronics to electronics. In addition, using a similar approach to the preparation of high-quality graphene layers via $\mathrm{CVD},{ }^{15}$ large scalable single-crystalline $\mathrm{h}-\mathrm{BN}$ films have recently been developed that can be practically utilized as a semiconductor growth substrate. ${ }^{16,17}$

${ }^{1}$ Department of Physics and Astronomy, Institute of Applied Physics, and Research Institute of Advanced Materials, Seoul National University, Seoul, Korea and ${ }^{2}$ Department of Materials Science and Engineering, Research Institute of Advanced Materials, Seoul National University, Seoul, Korea

Correspondence: Professor G-C Yi, Department of Physics and Astronomy, Institute of Applied Physics, and Research Institute of Advanced Materials, Seoul National University, Seoul 08826, Korea.

E-mail: gcyi@snu.ac.kr

Received 3 February 2017; revised 6 April 2017; accepted 27 April 2017 

Electrochemica
delamination

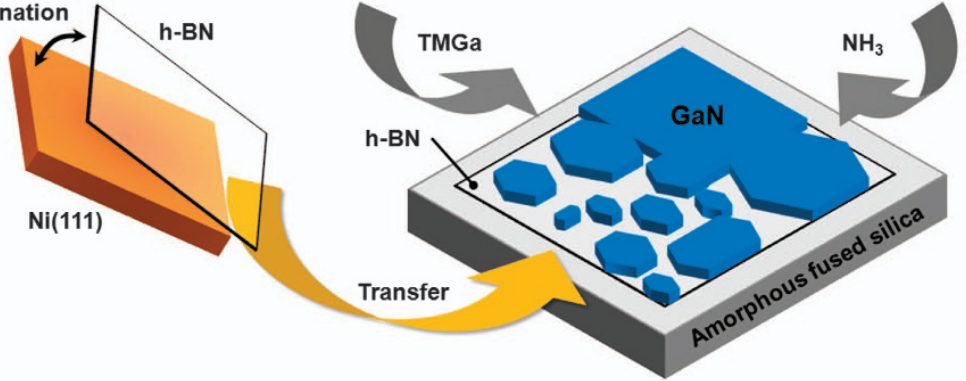

b

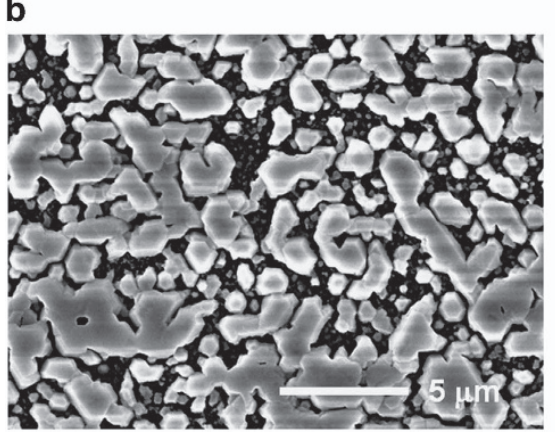

C

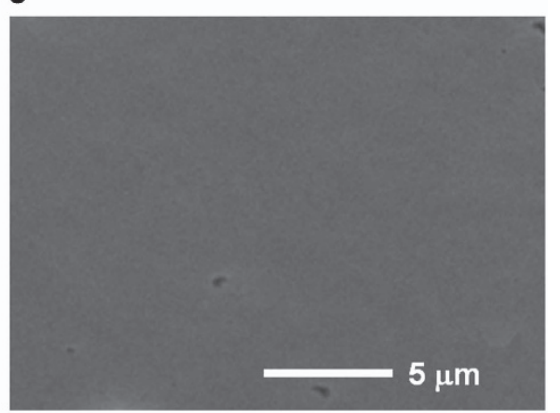

Figure 1 Growth of GaN films on transferable chemical vapor deposition (CVD)-grown hexagonal boron nitride (h-BN). (a) Schematic illustration of the CVDgrown h-BN transfer from $\mathrm{Ni}(111)$ to amorphous fused silica substrates and the gallium nitride (GaN) layer growth using metal-organic chemical vapor deposition (MOCVD). Field emission scanning electron microscopy (FE-SEM) images of (b) high-density discrete GaN islands that are almost merged into GaN microstructures with sizes of a few microns, and (c) fully coalescent GaN films grown on CVD-grown h-BN.

\section{MATERIALS AND METHODS}

Single-crystalline h-BN layers were synthesized on single-crystal $\mathrm{Ni}(111)$ with an area of $1 \times 1 \mathrm{~cm}$ by CVD, and served as GaN layer growth substrates, as shown in Figure 1a. The h-BN layers were readily lifted off from the Ni(111) using an electrochemical delamination technique. ${ }^{17}$ Because this technique does not require any $\mathrm{Ni}(111)$ substrate etching, h-BN layers can be repeatedly grown on the same $\mathrm{Ni}(111)$ substrate. In addition, when h-BN layers were prepared in a transferable form, they could serve as an intermediate layer for the growth of semiconductors, thus allowing arbitrary substrates to be used, including largesize and low-cost metal or amorphous substrates. In this study, the freestanding h-BN layers were transferred onto amorphous silica substrates before GaN layer growth, where this supporting substrate has no epitaxial relationship with GaN but has high thermal and chemical tolerance under the GaN growth conditions. The typical thickness of the CVD-grown h-BN was measured to be $3-6 \mathrm{~nm} .{ }^{17}$

The GaN layers were deposited on the CVD-grown h-BN layers using metalorganic CVD. Trimethylgallium and $\mathrm{NH}_{3}$ were used as the precursors, and the V/III ratio was maintained at 2000-4000 during the growth process. Before the GaN layer growth, the substrate was heated to $1100^{\circ} \mathrm{C}$ for 3-20 min with $\mathrm{H}_{2}$ gas only. Then, the temperature was decreased to $540-600{ }^{\circ} \mathrm{C}$ to grow a $\mathrm{GaN}$ nucleation layer. After 3 min of $\mathrm{GaN}$ nucleation layer growth, epitaxial GaN layers were grown at a higher temperature in the $1000-1100{ }^{\circ} \mathrm{C}$ range. No additional intermediate layers or substrate treatments were employed for $\mathrm{GaN}$ layer growth on CVD-grown h-BN. Field emission scanning electron microscopy was used for morphological analyses. The structural properties of the GaN layers grown on h-BN were measured using XRD and TEM. The planview and cross-sectional images of the GaN layers were obtained using a focused ion beam.

\section{RESULTS AND DISCUSSION}

The morphology of the GaN layers on CVD-grown h-BN layers was examined using field emission scanning electron microscopy. Figure $1 \mathrm{~b}$ shows that uniformly distributed $\mathrm{GaN}$ islands were grown over the entire CVD-grown h-BN, even though we did not employ any $\mathrm{ZnO}$ or $\mathrm{AlN}$ intermediate layers that have previously been used as an intermediate layer for $\mathrm{GaN}$ thin film growth on 2D layered materials. The diameter of discrete $\mathrm{GaN}$ islands was in the range of a few hundred nanometers, and the islands were already partially coalescent to form few-micron-size interconnecting GaN microstructures. Furthermore, by increasing the growth time, these discrete $\mathrm{GaN}$ microstructures were fully merged to form continuous $\mathrm{GaN}$ films with a flat surface morphology, as shown in Figure 1c. The direct growth of GaN layers on amorphous fused silica substrates typically yields rough and irregular surface morphologies because of the lack of an epitaxial relationship, suggesting that homogeneous GaN layers can be grown on CVD-grown h-BN without the use of single-crystal substrates underlying the h-BN layers. In addition, CVD-grown h-BN films were found to be suitable for the growth of semiconductors with high density, presumably because of the atomic cliffs inherent in the CVDgrown h-BN. As confirmed in our previous reports, ${ }^{17}$ atomic force microscopy and plan-view TEM results clearly show many naturally formed step edges in the CVD-grown h-BN films with nanometerscale spatial distances, with surfaces different from those of pristine h-BN layers prepared by mechanical exfoliation from h-BN powders.

We confirmed the effects of h-BN step edges or cliffs by growing GaN layers on mechanically exfoliated h-BN flakes (see Supplementary Figure S1). As the mechanical exfoliation technique enables a thin h-BN lamina to be peeled off with little surface damage, pristine h-BN can be prepared almost free of atomic cliffs. Meanwhile, unlike the growth features of GaN layers on CVD-grown h-BN, only one or two $\mathrm{GaN}$ microstructures could be grown on mechanically exfoliated h-BN flakes. Although we observed that GaN layers could be deposited uniformly on these pristine h-BN flakes at a low growth temperature of $500-600{ }^{\circ} \mathrm{C}$, the desorption of GaN layers occurred easily during the high-temperature growth process, where the high-temperature growth was crucial for obtaining highly crystalline GaN layers. The low-temperature GaN buffer layer plays a critical role in growing 
highly crystalline GaN films on conventional substrates such as $\mathrm{Al}_{2} \mathrm{O}_{3}$ by offering a high density of nucleation sites. However, for the growth of $\mathrm{GaN}$ on pristine 2D layered materials of graphene and h-BN, highdensity growth could not be achieved, even at low temperatures. This occurred because the $s p^{2}$-bonded 2D films are known to be free of dangling bonds, resulting in an extremely low chemical reactivity compared with that of the typical bulk substrates such as $\mathrm{Al}_{2} \mathrm{O}_{3}$.

To resolve this problem, we used a simple plasma treatment on the h-BN flake. The plasma etching process generates many atomic cliffs on h-BN. Subsequently, fully coalescent GaN layers could be grown on the plasma-treated h-BN flakes when using the low-temperature GaN buffer layer, presumably because the enhanced chemical reactivity of h-BN by atomic cliffs suppressed the desorption of the lowtemperature GaN buffer layer, thus providing sufficient nucleation islands to grow homogeneous GaN layers at higher temperatures. The position controls of one-dimensional semiconductor nanostructures on h-BN and graphene have also previously been examined using this preferable growth feature on plasma-treated 2D films. ${ }^{18,19}$ More importantly, as confirmed by our TEM measurements, the GaN layers exhibited identical crystal orientations to those of the h-BN substrate, indicating GaN heteroepitaxy on h-BN (see Supplementary Figure S2). This semiconductor growth on CVD-grown and plasma-treated 2D films suggests that the problems of low growth yields on 2D substrates can eventually be solved when 2D substrates exhibit a high number of atomic cliffs.

The structural properties of GaN films grown on CVD-grown h-BN layers were examined using XRD measurements. For $\theta-2 \theta$ scan results, we observed only two dominant XRD diffraction peaks at $34.57^{\circ}$ and $72.91^{\circ}$ in the $20^{\circ}-80^{\circ}$ measurement range corresponding to the (0002) and (0004) orientations of wurtzite $\mathrm{GaN}$, respectively (Figure 2a). No additional XRD peaks from the extremely thin h-BN layers and the amorphous fused silica supporting substrates were measured. The inset in Figure 2a shows the rocking curves of the GaN (0002) peak, where the full width at half maximum value of the rocking curve was measured to be $2.37^{\circ}$. Although the full width at half maximum value was slightly larger than that of the GaN films grown on CVD-grown graphene films, ${ }^{20}$ the results of both the $\theta-2 \theta$ scan and the rocking curve suggest the preferable $c$-axis alignment of the GaN films grown on CVD-grown h-BN. Furthermore, as shown in Figure $2 \mathrm{~b}$, azimuthal $(\phi)$-scans of the $\mathrm{GaN}(10 \overline{1} 2)$ direction exhibited diffraction peaks with sixfold symmetry. We also investigated the single crystallinity of the GaN layers grown on CVD-grown h-BN by using electron backscatter diffraction (see Supplementary Figure S3).

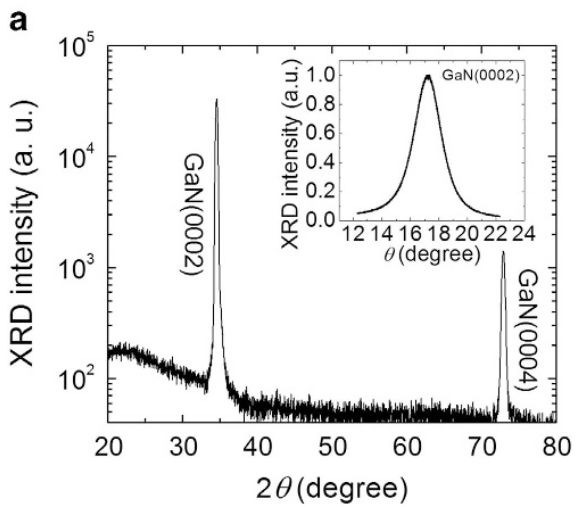

These results all strongly suggest that single-crystalline GaN films were grown on h-BN.

The microstructural properties of the GaN films grown on CVDgrown h-BN were further investigated by TEM. Because of the weak bonding between h-BN and amorphous fused silica substrates, we could easily detach the GaN film to prepare a TEM specimen. For plan-view TEM analysis, the GaN films were selectively thinned using a focused ion beam. Figure 3a shows a bright-field image of the planview TEM specimen. Although severe in-plane misorientations, such as a high-angle grain boundary, have commonly been observed in GaN films grown on polycrystalline CVD-grown $2 \mathrm{D}$ films, ${ }^{21}$ there were no distinguishable lines with high contrast in the bright-field image. Furthermore, the corresponding selected area electron diffraction (SAED) pattern in Figure $3 \mathrm{~b}$ exhibits good sixfold symmetry, in agreement with the in-plane $\mathrm{XRD}$ measurements, suggesting the improved crystallinity of the GaN films grown on single-crystal 2D substrates. Nevertheless, as shown in Figure 3c, the diffraction spot split of the SAED pattern became wider when we applied a larger aperture, indicating that the GaN film grown on CVD-grown h-BN possessed minor in-plane misorientations of $\mathrm{GaN}$ grains. As determined by $4.5-\mu \mathrm{m}$-aperture SAED patterns obtained from different regions of the plan-view specimen, the broadening angles were $6^{\circ}$ or smaller.

Direct observation of the low-angle grain boundaries in the $\mathrm{GaN}$ films was carried out using a plan-view two-beam dark-field (DF) image. Figure $3 \mathrm{~d}$ clearly shows the formation of a dislocation core with the low-angle grain boundaries as indicated by the arrows. The DF image was obtained with $g=[11 \overline{2} 0]$ to clearly observe the threading dislocations; ${ }^{22}$ many dislocations can be generated at the grain boundary regions to alleviate the stress and strain occurring during the coalescence of the slightly misaligned $\mathrm{GaN}$ grains. The lateral grain sizes that were found to be free of long connecting dislocation lines were estimated to be $200-1000 \mathrm{~nm}$, and this length scale agreed with the image obtained with the smaller aperture size, as shown in Figure 3b, that showed clear sixfold symmetry in the SAED pattern. In addition to the dislocation lines bounded to the low-angle boundaries, individual threading dislocations were observed in the grain boundaryfree region, as revealed by spots, small lines and a mixture of spots and lines (Figure 3e). The density of these individual dislocations was $1.0-4.4 \times 10^{9} \mathrm{~cm}^{-2}$, comparable to those of the GaN layers epitaxially grown on h-BN-coated $c-\mathrm{Al}_{2} \mathrm{O}_{3}$ substrates or on single-crystalline mechanically exfoliated graphene layers. ${ }^{23,24}$

We also investigated the microstructural behaviors of the GaN films grown on CVD-grown h-BN using cross-sectional TEM analysis, b

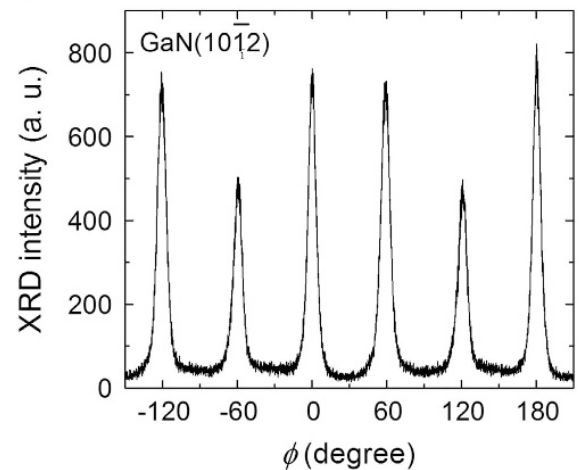

Figure 2 X-ray diffraction (XRD) results of the gallium nitride (GaN) films grown on chemical vapor deposition (CVD)-grown hexagonal boron nitride (h-BN). (a) The $\theta-2 \theta$ scan with an inset showing a rocking curve of the GaN(0002) peak. (b) The $\phi$-scan of the GaN(1012) direction. 
a

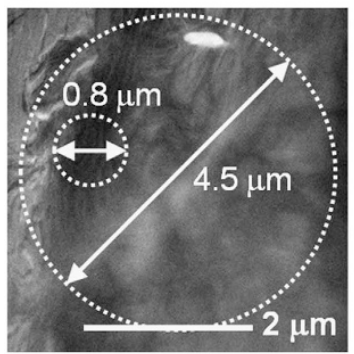

d

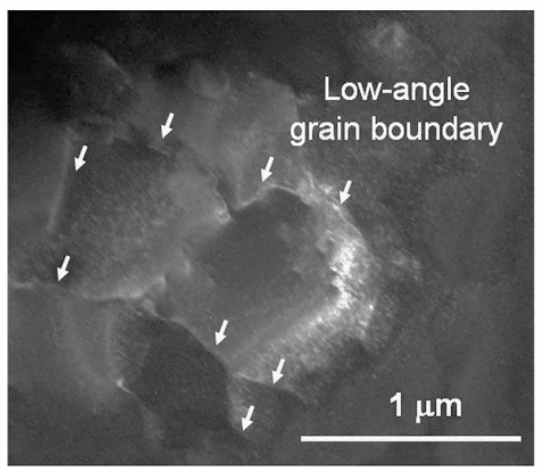

b

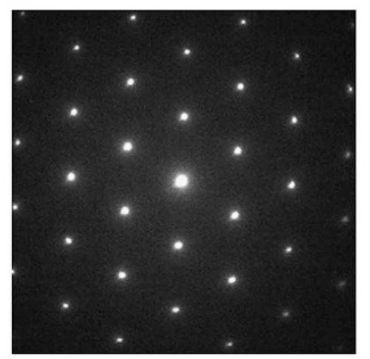

e

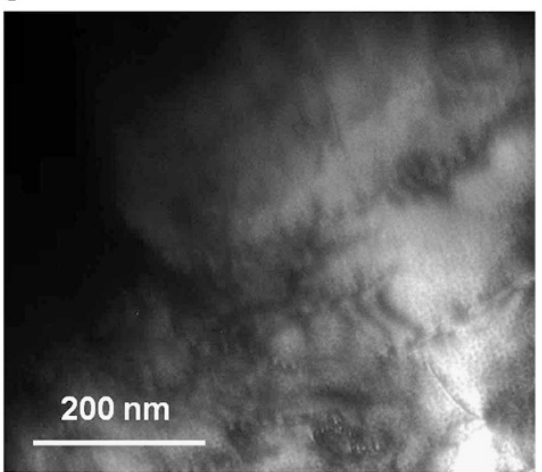

Figure 3 Plan-view transmission electron microscopy (TEM) analysis of the gallium nitride (GaN) films grown on chemical vapor deposition (CVD)-grown hexagonal boron nitride ( $h-B N$ ). Selected area electron diffraction (SAED) patterns were obtained from (a) bright-field (BF) image of the GaN films with aperture sizes of (b) $0.8 \mu \mathrm{m}$ and (c) $4.5 \mu \mathrm{m}$. (d) Two-beam dark-field (DF) image taken with $\mathrm{g}=[11 \overline{2} 0]$ and (e) its magnified image.

a

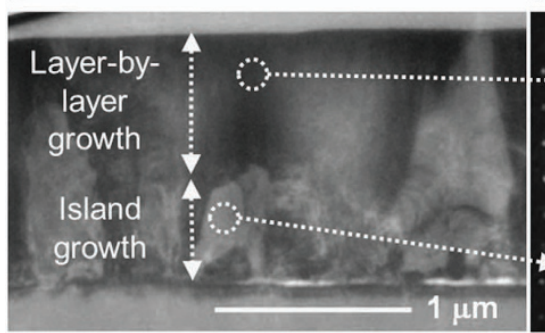

d

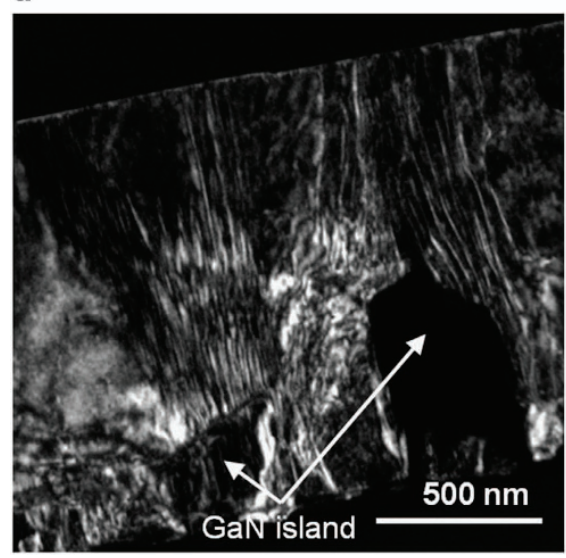

b
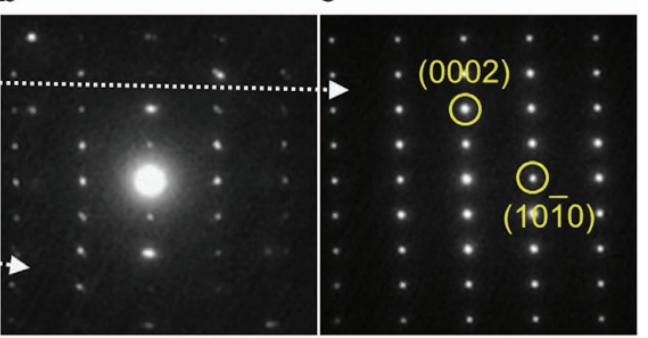

e

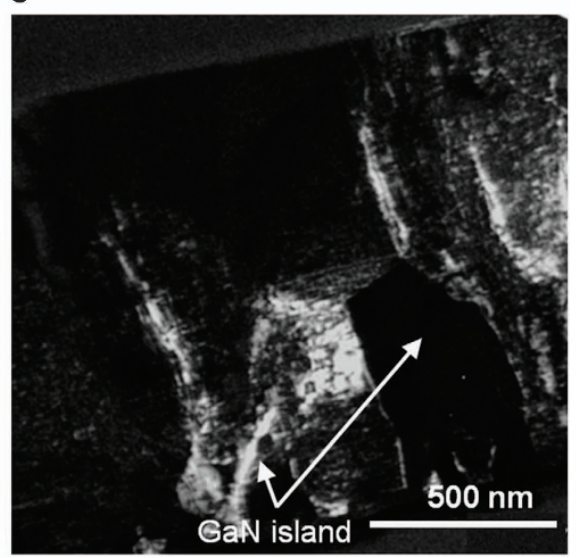

Figure 4 Cross-sectional transmission electron microscopy (TEM) analysis of gallium nitride (GaN) films grown on chemical vapor deposition (CVD)-grown hexagonal boron nitride (h-BN). (a) Cross-sectional bright-field (BF) image. Selected area electron diffraction (SAED) patterns were obtained from (b) the lower part of GaN islands and (c) the upper part of fully coalescent GaN films. Cross-sectional two-beam dark-field (DF) images taken with (d) $g=[0002]$ and (e) $g=[10 \overline{1} 0]$. 
allowing us to scan the entire GaN microstructure. The cross-sectional bright-field TEM image in Figure 4a shows that the layer-by-layer grown $\mathrm{GaN}$ layers were formed on the 400-600-nm-high GaN islands, consistent with the field emission scanning electron microscopy results. The total thickness of the GaN layers was found to be $1300 \mathrm{~nm}$. The SAED pattern of the GaN islands exhibited a regular spot array of hexagonal wurtzite $\mathrm{GaN}$ (Figure 4b). As the lowtemperature growth resulted in the dominant $\mathrm{GaN}$ crystals with a cubic zincblende structure, ${ }^{25,26}$ this result suggests that larger GaN islands can be grown on h-BN during the high-temperature growth. We also observed split spots in the SAED pattern of the GaN island, indicating slightly misaligned GaN crystal orientations. However, the upper part of the GaN film showed a well-ordered SAED pattern with single orientation spots, representing the formation of highly crystalline $\mathrm{GaN}$ layers following the coalescence of $\mathrm{GaN}$ islands (Figure 4c).

We also obtained two-beam DF cross-sectional TEM images of the $\mathrm{GaN}$ films grown on CVD-grown h-BN layers that are useful for determining the dislocation types in GaN. Because of the different Burgers vectors of screw-type and edge-type dislocations in hexagonal $\mathrm{GaN}$, pure screw- and edge-type dislocations are only visible under $g=[0002]$ and [1010], respectively, whereas mixed-type dislocations can be seen in both reciprocal lattice vectors. ${ }^{27}$ Figure $4 \mathrm{~d}$ shows a $\mathrm{g}=[0002]$ two-beam image of the $\mathrm{GaN}$ layers grown on h-BN, revealing many screw-type dislocation lines almost parallel to the $c$ axis. Interestingly, these vertical dislocation lines were predominantly observed above the GaN islands rather than in the island-free region. Note that we confirmed the presence of $\mathrm{GaN}$ islands by using the corresponding bright-field image, even though some $\mathrm{GaN}$ islands exhibited dark contrast in the DF images (see Supplementary Figure S4). In the $g=[10 \overline{1} 0]$ two-beam image, we also observed vertical lines of pure edge- and mixed-type dislocations above or near the $\mathrm{GaN}$ islands, but their density was much lower than that of the screw-type dislocations (Figure 4e). Figure 4e also shows some lateral lines of pure edge-type dislocations that presumably resulted from the lateral growth of $\mathrm{GaN}$ layers for the merging of the $\mathrm{GaN}$ islands. However, the cross-sectional DF TEM results show that most of the vertical threading dislocations in $\mathrm{GaN}$ films grown on $\mathrm{h}-\mathrm{BN}$ are pure screw-type dislocations with limited numbers of mixed-type dislocations, whereas pure edge-type dislocations were found to be dominant in the previous study of $\mathrm{GaN}$ on sapphire substrates. ${ }^{28}$

A relatively large number of screw-type dislocations have been observed in previous studies of $\mathrm{GaN}$ films grown on 2D layered materials such as h-BN and graphene. ${ }^{23,24}$ Nevertheless, we found that these screw-type dislocation lines originated from the GaN islands, where the morphological evolution of $\mathrm{GaN}$ films grown on h-BN strongly suggested that $\mathrm{GaN}$ islands should initially grow on h-BN atomic cliffs. More interestingly, the morphological and microstructural behaviors of the GaN films grown on 2D substrates were found to agree well with the theoretical prediction regarding crystal growth on a facet of a perfect crystal. This theory proposes that when the growth conditions are subject to low supersaturations on perfect crystal facets, screw-type dislocations can play a critical role in crystal growth because cliffs of atoms driven by these dislocations can serve as a new crystal edge for larger growth exceeding a certain critical size. ${ }^{29,30}$ Almost identical to the predictions of this theory, we demonstrated that GaN layers did not grow on h-BN unless the h-BN substrates had a high number of atomic cliffs. The predominance of screw-type dislocations in the GaN island regions also provided strong evidence that semiconductor growth on $2 \mathrm{D}$ films can be directly correlated to the crystal growth mechanism driven by atomic cliffs. Furthermore, the GaN films grown on single-crystal CVD-grown h-BN exhibited many low-angle grain boundaries that were presumably because of these screw-type dislocations. Typically, the high interfacial energy between $\mathrm{GaN}$ and the heterogeneous substrate yields low-angle grain boundaries in GaN layers. Furthermore, screw-type dislocations can twist the inplane orientations of $\mathrm{GaN}$ grains. However, we believe that the structural imperfections in GaN films grown on CVD h-BN could be further improved by using similar approaches to those used to grow higher crystalline GaN layers on other heterogeneous substrates, that is, by increasing thickness or epitaxial lateral overgrowth. ${ }^{31,32}$

\section{CONCLUSION}

To conclude, the heteroepitaxial growth of GaN films on CVD-grown $\mathrm{h}$-BN layers was demonstrated in which the generation of a high number of atomic cliffs in the h-BN substrate was the key process in fabricating these GaN/h-BN heterostructures. Promisingly, CVDgrown h-BN already exhibited many atomic cliffs, thus enabling the growth of high-density $\mathrm{GaN}$ islands that merged to form a flat, homogeneous film. In addition, nearly single-crystal GaN layers were grown directly on CVD-grown h-BN without the use of other singlecrystal substrates. The clear observation of high-density epitaxial growth of GaN layers on large-size scalable single-crystal CVD-grown h-BN opens a pathway to novel applications of h-BN in the development of a range of inorganic semiconductor devices.

\section{CONFLICT OF INTEREST}

The authors declare no conflict of interest.

\section{ACKNOWLEDGEMENTS}

This work was supported by the Global Research Laboratory Program through the National Research Foundation of Korea (NRF) funded by the Ministry of Science, ICT and Future Planning (NRF-2015K1A1A2033332 and NRF-2015R1A 2A1A15055406) and Research Cooperation Program of Seoul National University (SNU) and the Yonsei University that was run by SNU in 2015.

\section{PUBLISHER'S NOTE}

Springer Nature remains neutral with regard to jurisdictional claims in published maps and institutional affiliations.

1 Fan, Z., Razavi, H., Do, J.-W., Moriwaki, A., Ergen, O., Chueh, Y.-L., Leu, P. W., Ho, J. C., Takahashi, T., Reichertz, L. A., Neale, S., Yu, K., Wu, M., Ager, J. W. \& Javey, A. Three-dimensional nanopillar-array photovoltaics on low-cost and flexible substrates. Nat. Mater. 8, 648-653 (2009).

2 Rogers, J. A., Someya, T. \& Huang, Y. Materials and mechanics for stretchable electronics. Science 327, 1603-1607 (2010).

3 Harris, K. D., Elias, A. L. \& Chung, H.-J. Flexible electronics under strain: a review of mechanical characterization and durability enhancement strategies. J. Mater. Sci. 51, 2771-2805 (2016).

4 Chung, K., Lee, C.-H. \& Yi, G.-C. Transferable GaN layers grown on ZnO-coated graphene layers for optoelectronic devices. Science 330, 655-657 (2010).

5 Lee, C.-H., Kim, Y.-J., Hong, Y. J., Jeon, S.-R., Bae, S., Hong, B. H. \& Yi, G.-C. Flexible inorganic nanostructure light-emitting diodes fabricated on graphene films. Adv. Mater. 23, 4614-4619 (2011).

6 Gupta, P., Rahman, A. A., Hatui, N., Parmar, J. B., Chalke, B. A., Bapat, R. D., Purandare, S. C., Deshmukh, M. M. \& Bhattacharya, A. Free-standing semipolar IIInitride quantum well structures grown on chemical vapor deposited graphene layers. Appl. Phys. Lett. 103, 181108 (2013).

7 Kim, J., Bayram, C., Park, H., Cheng, C.-W., Dimitrakopoulos, C., Ott, J. A., Reuter, K. B., Bedell, S. W. \& Sadana, D. K. Principle of direct van der Waals epitaxy of singlecrystalline films on epitaxial graphene. Nat. Commun. 5, 4836 (2014).

8 Choi, D., Choi, M.-Y., Choi, W. M., Shin, H.-J., Park, H.-K., Seo, J.-S., Park, J., Yoon, S.-M., Chae, S. J., Lee, Y. H., Kim, S.-W., Choi, J.-Y., Lee, S. Y. \& Kim, J. M. Fully rollable transparent nanogenerators based on graphene electrodes. Adv. Mater. 22, 2187-2192 (2010)

9 Park, W. I., Lee, J. M., Lee, D. H., Yi, G.-C. in Semiconductor Nanostructures for Optoelectronic Devices: Processing, Characterization and Application ed. Yi, G.-C Ch. 6, 167-195 Springer: Heidelberg, Germany (2012). 
10 Munshi, A. M. \& Weman, H. Advances in semiconductor nanowire growth on graphene. Phys. Status Solidi RRL 7, 713-726 (2013).

11 Watanabe, K., Taniguchi, T. \& Kanda, H. Direct-bandgap properties and evidence for ultraviolet lasing of hexagonal boron nitride single crystal. Nat. Mater. 3, 404-409 (2004).

12 Song, L., Ci, L., Lu, H., Sorokin, P. B., Jin, C., Ni, J., Kvashnin, A. G., Kvashnin, D. G., Lou, J., Yakobson, B. I. \& Ajayan, P. M. Large scale growth and characterization of atomic hexagonal boron nitride layers. Nano Lett. 10, 3209-3215 (2010).

13 Lee, G.-H., Yu, Y.-J., Lee, C., Dean, C., Shepard, K. L., Kim, P. \& Hone, J. Electron tunneling through atomically flat and ultrathin hexagonal boron nitride. Appl. Phys. Lett. 99, 243114 (2011)

14 Lin, Y. \& Connell, J. W. Advances in 2D boron nitride nanostructures: nanosheets, nanoribbons, nanomeshes, and hybrids with graphene. Nanoscale 4 6908-6939 (2012).

15 Lee, J.-H., Lee, E. K., Joo, W.-J., Jang, Y., Kim, B.-S., Lim, J. Y., Choi, S.-H., Ahn, S. J., Ahn, J. R., Park, M.-H., Yang, C.-W., Choi, B. L., Hwang, S.-W. \& Whang, D. Waferscale growth of single-crystal monolayer graphene on reusable hydrogen-terminated germanium. Science 344, 286-289 (2014)

16 Lu, G., Wu, T., Yuan, Q., Wang, H., Wang, H., Ding, F., Xie, X. \& Jiang, M. Synthesis of large single-crystal hexagonal boron nitride grains on $\mathrm{Cu}-\mathrm{Ni}$ alloy. Nat. Commun. 6 , 6160 (2015).

17 Oh, H., Jo, J., Tchoe, Y., Yoon, H., Lee, H. H., Kim, S.-S., Kim, M., Sohn, B.-H. \& Yi, G.-C. Centimeter-sized epitaxial h-BN films. NPG Asia Mater. 8, e330 (2016).

$18 \mathrm{Kim}$, Y.-J., Yoo, H., Lee, C.-H., Park, J. B., Baek, H., Kim, M. \& Yi, G.-C. Position- and morphology-controlled $\mathrm{ZnO}$ nanostructures grown on graphene layers. Adv. Mater. 24 5565-5569 (2012).

19 Oh, H., Hong, Y. J., Kim, K.-S., Yoon, S., Baek, H., Kang, S.-H., Kwon, Y.-K., Kim, M. $\& \mathrm{Yi}, \mathrm{G}$.-C. Architectured van der Waals epitaxy of $\mathrm{ZnO}$ nanostructures on hexagonal BN. NPG Asia Mater. 6, e145 (2014).

20 Chung, K., Park, S. I., Baek, H., Chung, J. S. \& Yi, G.-C. High-quality GaN films grown on chemical vapor-deposited graphene films. NPG Asia Mater. 4, e24 (2012).

21 Yoo, H., Chung, K., Park, S. I., Kim, M. \& Yi, G.-C. Microstructural defects in GaN thin films grown on chemically vapor-deposited graphene layers. Appl. Phys. Lett. 102, 051908 (2013).

22 Follstaedt, D. M., Missert, N. A., Koleske, D. D., Mitchell, C. C. \& Cross, K. C. Plan-view image contrast of dislocations in GaN. Appl. Phys. Lett. 83, 4797-4799 (2003).

23 Yoo, H., Chung, K., Choi, Y. S., Kang, C. S., Oh, K. H., Kim, M. \& Yi, G.-C. Microstructures of GaN thin films grown on graphene layers. Adv. Mater. 24 515-518 (2012).
24 Kobayashi, Y., Kumakura, K., Akasaka, T. \& Makimoto, T. Layered boron nitride as a release layer for mechanical transfer of GaN-based devices. Nature 484, 223-227 (2012).

25 Wu, X. H., Kapolnek, D., Tarsa, E. J., Heying, B., Keller, S., Keller, B. P., Mishra, U. K., DenBaars, S. P. \& Speck, J. S. Nucleation layer evolution in metal-organic chemical vapor deposition grown GaN. Appl. Phys. Lett. 68, 1371-1373 (1996).

26 Vennegues, P., Beaumont, B., Vaille, M. \& Gibart, P. Microstructure of GaN epitaxial films at different stages of the growth process on sapphire (0001). J. Cryst. Growth 173, 249-259 (1997).

27 Hirsch, P. B., Howie, A., Nicholson, R. B., Pashley, D. W. \& Whelan, M. J. Electron Microscopy of Thin Crystals, Krieger: New York (1977).

28 Rouviere, J. L., Arlery, M., Daudin, B., Feuillet, G. \& Briot, O. Transmission electron microscopy structural characterisation of $\mathrm{GaN}$ layers grown on (0001) sapphire. Mat. Sci. Eng. B Adv. Funct. Solid State Mater. 50, 61-71 (1997).

29 Burton, W. K., Cabrera, N. \& Frank, F. C. Role of dislocations in crystal growth. Nature 163, 398-399 (1949).

30 Burton, W. K., Cabrera, N. \& Frank, F. C. The growth of crystals and the equilibrium structure of their surfaces. Philos. Trans. R. Soc. London A 243, 299-358 (1951).

31 Kapolnek, D., Wu, X. H., Heying, B., Keller, S., Keller, B. P., Mishra, U. K., DenBaars, S. P. \& Speck, J. S. Structural evolution in epitaxial metalorganic chemical vapor deposition grown GaN films on sapphire. Appl. Phys. Lett. 67, 1541-1543 (1995).

32 Nakamura, S. The roles of structural imperfections in InGaN-based blue light-emitting diodes and laser diodes. Science 281, 956-961 (1998).

This work is licensed under a Creative Commons Attribution 4.0 International License. The images or other third party material in this article are included in the article's Creative Commons license, unless indicated otherwise in the credit line; if the material is not included under the Creative Commons license, users will need to obtain permission from the license holder to reproduce the material. To view a copy of this license, visit http:// creativecommons.org/licenses/by/4.0/

(C) The Author(s) 2017

Supplementary Information accompanies the paper on the NPG Asia Materials website (http://www.nature.com/am) 